\title{
Corrigendum to "Present status of Medical Physics practice in Mexico: An occupational analysis" [Phys. Med. 76 (2020) 55-61]
}

\author{
Diana García-Hernández ${ }^{\mathrm{a}}$, Xóchitl López-Rendón ${ }^{\mathrm{b}}$, Mariana Hernández-Bojórquez ${ }^{\mathrm{c}, \mathrm{h}}$, \\ José Alfredo Herrera-González ${ }^{\mathrm{d}}$, Orlando Enrique Soberanis-Domínguez ${ }^{\mathrm{e}}$, \\ Suemi Alejandra González-Azcorra ${ }^{\mathrm{f}}$, Juan Pablo Cruz-Bastida ${ }^{\mathrm{g}}$,* \\ ${ }^{a}$ Unidad de Especialidades Médicas de Oncología, Servicios de Salud de Zacatecas, Av. Barones \#3, Ejidal, 98613 Guadalupe, Zacatecas, Mexico \\ ${ }^{\mathrm{b}}$ Departamento de Neuroimagen, Instituto Nacional de Neurología y Neurocirugía, Av. Insurgentes Sur \#3877, La Fama, 14269, Tlalpan, Mexico City, Mexico \\ ${ }^{\mathrm{c}}$ Departamento de Radioterapia, The American British Cowdray Medical Center, Sur 136 \#116, Las Americas, 01120, Alvaro Obregon, Mexico City, Mexico \\ ${ }^{\mathrm{d}}$ Unidad de Radioneurocirugía, Instituto Nacional de Neurología y Neurocirugía, Av. Insurgentes Sur \#3877, La Fama, 14269, Tlalpan, Mexico City, Mexico \\ e Departamento de Medicina Nuclear, Centro Oncológico Privado, Calle 21 \#588, Maya, 97134 Merida, Yucatan, Mexico \\ ${ }^{\mathrm{f}}$ Departamento de Radioncología, Hospital Agustín O'Horán, Av. Itzaes s/n y Av, Centro Jacinto Canek, 97000 Merida, Yucatan, Mexico \\ ${ }^{g}$ Department of Radiology, University of Chicago, 5841 South Maryland Avenue, MC2026 Chicago, IL, USA \\ ${ }^{\mathrm{h}}$ Escuela Superior de Física y Matemáticas, Instituto Politécnico Nacionals, Av. Instituto Politécnico Nacional s/n Edificio 9, San Pedro Zacatenco, 07738, Gustavo A. \\ Madero, Mexico City, Mexico
}

The authors regret that an error occurred in Table 1 of the abovementioned article.

Under the "Institution" heading in Table 1, it incorrectly states
"Universidad Autónoma de Sonora". The correct affiliation is "Universidad de Sonora".

The authors would like to apologise for any inconvenience caused.

DOI of original article: https://doi.org/10.1016/j.ejmp.2020.06.008.

* Corresponding author.

E-mail address: cruzbastida@uchicago.edu (J.P. Cruz-Bastida). 